\title{
On the variability of the Charnock constant and the functional dependence of the drag coefficient on wind speed
}

\author{
Part II Observations
}

\author{
John A. T. Bye · Jörg-Olaf Wolff · Karsten A. Lettmann
}

Received: date / Accepted: date

\begin{abstract}
An analytical expression for the $10 \mathrm{~m}-\operatorname{drag}_{21}$ law in terms of the $10 \mathrm{~m}$ wind speed at the maximum in ${ }_{22}$ the $10 \mathrm{~m}$ drag coefficient, and the Charnock constant is 23 presented, which is based on the results obtained from 24 a model of the air-sea interface derived in Bye et al. 25 (2010). This drag law is almost independent of wave age 26 and over the mid-range of wind speeds $\left(5-17 \mathrm{~ms}^{-1}\right)_{27}$ is very similar to the drag law based on observed data 28 presented in Foreman and Emeis (2010). The linear fit 29 of the observed data, which incorporates a constant into ${ }_{30}$ the traditional definition of the drag coefficient is shown 31 to arise to first-order as a consequence of the momen- 32 tum exchange across the air-sea boundary layer brought ${ }_{33}$ about by wave generation and spray production which 34 are explicitly represented in the theoretical model.
\end{abstract}

Keywords Air-sea momentum exchange $\cdot$ Charnock ${ }^{36}$ constant $\cdot$ inertial coupling

\section{Introduction}

The purpose of this paper is to bring together the re- 42 sults of several studies of the drag coefficient in the air- 43

\section{J. A. T. Bye}

School of Earth Sciences, The University of Melbourne, Vic- ${ }^{45}$ toria 3010, Australia

Tel.: +61383440046

Fax: +61 383447761

E-mail: jbye@unimelb.edu.au

J.-O. Wolff

ICBM, Carl-von-Ossietzky Universität Oldenburg, Postfach ${ }^{50}$ 2053, 26111 Oldenburg, Germany

E-mail: wolff@icbm.de

K. A. Lettmann

ICBM, Carl-von-Ossietzky Universität Oldenburg, Postfach ${ }^{54}$ 2053, 26111 Oldenburg, Germany

E-mail: lettmann@icbm.de sea boundary layer presented in a recent observational paper (Foreman and Emeis 2010), and the theoretical predictions for the drag coefficient presented in Bye et al. (2010). At the time of writing of the latter paper the analysis of the observational results was not available. Here we show the basic agreement of the theoretical model with the observations, and also discuss the conclusions of the theoretical model as a description of the various sea states that are encountered.

This paper is essentially Part 2 of Bye et al. (2010), which for brevity we call Part 1. A full historical summary of the programmes on which the observed drag law is based can be found in Foreman and Emeis (2010), and equally the background analysis for the theoretical drag law can be found in Bye et al. (2010). An explicit representation of the theoretical results for the $10 \mathrm{~m}$ drag law and the Charnock constant in Part 1, however is given for the first time in this paper.

In Section 2 the physical processes on which the theoretical model is based are discussed, and in Section 3 the analytical expression for the $10 \mathrm{~m}$-drag coefficient based on the results in Part 1, which are presented in the Appendix, is given. An important subsection (Section 3.1) introduces the use of the Charnock constant as a constraint on the prediction for the drag coefficient, derived from the observed wind and wave properties at the maximum of the $10 \mathrm{~m}$-drag coefficient. Section 3.2 demonstrates the key result that the drag law is almost independent of wave age.

In Section 4 the observations presented in Foreman and Emeis (2010) and also in a later paper by Andreas et al. (2012) are critically discussed, and it is shown that the theoretical model for the drag coefficient is a good representation of the observations in which the simple linear regression relation for the drag used in the observational study arises as a fundamental prop- 
${ }_{77} u_{\star}=\frac{\sqrt{K_{I}}}{R} u_{1}$ per is discussed in the Conclusion (Section 5).

\section{The physical processes} generation, which is represented by the relation,

$c_{0}=B u_{1}$ 3.2. The second process is frictional drag in the air-sea boundary layer, which is represented by the relation, erty. The complementary nature of the physical models 97 presented in Foreman and Emeis (2010) and in this pa- 98

There are three processes which govern the construc ${ }^{102}$ tion of the model for the $10 \mathrm{~m}$ drag law, $u_{\star}=\sqrt{K_{10}} \cdot u_{10}{ }^{103}$ where $u_{\star}$ is the friction velocity in air, $u_{10}$ is the $10 \mathrm{~m}^{104}$ wind speed and $K_{10}$ is the $10 \mathrm{~m}$ drag coefficient, pro- $^{105}$ posed in Bye et al. (2010). The first process is wave

where $c_{0}=g T / 2 \pi$ is the peak wave speed in which $g_{111}^{110}$ is the acceleration of gravity and $T$ is the peak wave period, and $u_{1}$ is the surface wind speed which occurs at the height, $z_{B}=1 /\left(2 k_{0}\right)$ where $k_{0}=g / c_{0}^{2}$ is the peak wave number, and $B$ is a wave generation parameter, which is determined by the nature of the wind field which generates the wave field as discussed in Section

where $K_{I}$ is the inertial drag coefficient ${ }^{1}$ and $R$ is ${ }^{119}$ frictional parameter which quantifies the relative con- $^{-121}$ tributions of the velocity shear in the wave boundary ${ }_{122}$ layer due to wave motion and turbulence. The wave- ${ }_{123}$ induced shear, which is due to the Lagrangian Stokes ${ }_{124}$ drift of the wave spectrum, is the difference between ${ }_{125}$ the Stokes drift at the sea surface and that at the bottom of the wave boundary layer which is zero, and the ${ }_{127}$ turbulent shear, which is due to the Eulerian velocity $y_{128}$ shear, is the difference between the frictional current ${ }_{129}$ at the sea surface and the surface current $\left(u_{2}\right)$ at the $\mathrm{I}_{130}$ bottom of the wave boundary layer. For $R<1$ the tur- $^{-131}$ bulent shear has the same sign as the wave shear, for $_{132}$ $R=1$, the ratio of turbulent/wave shear is zero, $\operatorname{and}_{133}$ for $R>1$ the turbulent shear is of opposite sign to the $\mathrm{e}_{134}$ wave shear indicating a return of momentum from the $\mathrm{e}_{135}$ ocean to the atmosphere by frictional drag, see Part 1.

The consideration of near surface shear in both fluids ${ }^{136}$ is a central feature of the model. The third process is

1 The inertial drag coefficient is defined by the drag rela-138 tion, $u_{\star}=\sqrt{K_{I}}\left(u_{1}-u_{2} / \epsilon\right)$ in terms of $u_{1}$ and the surface ${ }_{39}$ current speed, $u_{2}$, which occurs at the depth $1 /\left(2 k_{0}\right)$, where $\epsilon=\sqrt{\left(\rho_{1} / \rho_{2}\right)}$ in which $\rho_{1}$ and $\rho_{2}$ are respectively the density ${ }_{140}$ of air and water. $K_{I}$ is a property of the irrotational wave spectrum without recourse to frictional processes and field observations from the fully developed growing wind-wave sea ${ }^{141}$ indicate that $K_{I}=0.0015$ (Bye and Wolff 2008). the generation of spray which is represented through the relation,

$R=\frac{R_{0}}{1-a u_{\star}} \quad a>0$

in which $a$ is the spray parameter and $R_{0}$ is the friction parameter at very low friction velocities. This relation enables $R$ to be evaluated in terms of $u_{\star}$, and the increase in $R$ as $u_{\star}$ increases shows that the spray (and the associated processes of whitecapping and slip) essentially return momentum to the atmosphere. The use of (3) leads to drag laws in which there is a maximum in the $10 \mathrm{~m}$ drag coefficient.

The three parameters $\left(B, R_{0}\right.$ and $\left.a\right)$ were expressed in Part 1 in terms of three properties of the wavefield at the maximum in drag coefficient, namely the $10 \mathrm{~m}$ wind speed $\left(u_{10 m}\right)$, the drag coefficient maximum $\left(K_{10 m}\right)$ and the peak wave period $\left(T_{m}\right)$. Hence in the model on assuming these quantities to be known, there are no disposable constants. Note that the maximum friction velocity, $u_{\star m}=\sqrt{K_{10 m}} \cdot u_{10 m}$ and the maximum wave speed, $c_{0 m}=g T_{m} / 2 \pi$ follow from these three quantities.

\section{The 10m drag law}

The model has a universal character on the assumption that (3) realistically represents the spray/surface slip mechanism, and its application yields a 10m drag law which was shown to be almost independent of the wave generation parameter $(B)$. This was illustrated for two values $(B=0.66$ and $B=1.30)$ in Fig. 3 of Part 1 , from which it was concluded that the $10 \mathrm{~m}$ drag law was almost independent of wave age $\left(W=c_{0} / u_{\star}\right)$.

Here we build on the results of Part 1 by deriving an explicit relation for the $10 \mathrm{~m}$ drag law which illustrates this conclusion.

This procedure essentially requires a transformation of the physical results for which the natural vertical scale is the thickness of the wave boundary layer $\left(z_{B}\right)$ to the reference height of $10 \mathrm{~m}$ upon which the $10 \mathrm{~m}$ drag coefficient is defined. For a logarithmic velocity profile, this transformation yields,

$u_{10}=u_{1}+\frac{u_{\star}}{\kappa} \ln \frac{z_{10}}{z_{B}}$

where $z_{10}=10 \mathrm{~m}$ and $\kappa=0.4$ is von Kármán's constant, and in which from (1) and (2) $u_{1}=R u_{\star} / \sqrt{K_{I}}$ and $z_{B}=1 / 2\left(B R u_{\star}\right)^{2} /\left(g K_{I}\right)$. Hence,

$\frac{1}{\sqrt{K_{10}}}=\frac{R}{\sqrt{K_{I}}}-\frac{1}{\kappa} \ln \frac{\left(B R u_{\star}\right)^{2}}{2 g z_{10} K_{I}}$

On invoking (3) and differentiating (5) with respect to $u_{\star}$ assuming that $B$ is a constant, and then evaluating 
at the maximum in the $10 \mathrm{~m}$ drag coefficient $\left(K_{10 \mathrm{~m}}\right)_{186}$ after some algebra, we obtain,

$R_{m}=R_{0}+2 \frac{\sqrt{K_{I}}}{\kappa}$

187

188

$(6)_{189}$

190

in which $R_{m}$ is the friction parameter at $K_{10 m}$ (Bye et al. 2010). Note that $R_{m}>R_{0}$ indicating the return of momentum to the air which is occurring due to the production of spray. This innocent looking relation enables the $10 \mathrm{~m}$ drag law to be explicitly evaluated in ${ }^{194}$ terms of the sea state occurring at the drag coefficient maximum, namely the $10 \mathrm{~m}$ wind velocity $\left(u_{10 \mathrm{~m}}\right)$, the $10 \mathrm{~m}$ drag coefficient $\left(K_{10 \mathrm{~m}}\right)$ and the peak wave speed $\left(c_{0 m}\right)$, as is shown in the Appendix. The resulting uni- ${ }^{198}$ versal relation (A5) for $u_{10}$ in terms of $u_{\star}$ is,

$u_{10}=A(s) u_{\star}+s u_{10 m}$

where

$A(s)=\frac{2}{\kappa}\left\{\frac{X(s-1)}{(X-s)}-\ln \left[\frac{(X-1) s}{(X-s)}\right]\right\}$

in which $\kappa$ is von Kármán's constant, $s=u_{\star} / u_{\star m}$ and $X=1 /\left(a u_{\star m}\right)$, which may be inverted to yield the $10 \mathrm{~m}_{200}$ drag law,

$u_{\star}=\sqrt{K_{10 m}} \cdot u_{10}-\sqrt{K_{10 m}} \cdot A(s) u_{\star}$

In (8), $\left(-\sqrt{K_{10 m}} \cdot A(s) u_{\star}\right)$ may be regarded as an offset ${ }_{200}^{200}$ to a simple drag law in which at all wind speeds the drag $_{210}$ coefficient is equal to its maximum value. For $A(s)>0,_{211}$ this term reduces the drag coefficient as the wind speed $_{212}$ decreases.

3.1 The use of the Charnock constant in the specification of the drag law

The additional requirement that the value of the Charnock constant,

$\alpha_{0}=\frac{g z_{0}}{u_{\star}^{2}}$

$(9)_{221}$

where $z_{0}$ is the sea surface roughness length, agrees with observations, leads to a preferred choice for the value $2_{24}$ of $K_{10 m}$, which is not well defined by observation, $\operatorname{and}_{225}$ the restriction of the model to "aerodynamically" $\operatorname{rough}_{226}$ conditions which occur at the onset of wave growth at $u_{10}>3 \mathrm{~ms}^{-1}$ (Bye and Babanin 2009) implies that in ${ }^{227}$ the comparison with observations the Charnock con-228 stant should be evaluated from the theoretical modek 29 at this threshold wind speed, see Appendix.

The results in the Appendix assuming that $K_{10 m}=231$ $0.002, u_{10 m}=40 \mathrm{~ms}^{-1}$ and $T_{m}=17.6 \mathrm{~s}\left(c_{0 m}=27.6 \mathrm{~ms}_{\overline{232}}{ }^{-1}\right)$ lead to the following properties for the drag law: $X=233$ 5.15 (A2), $a=0.11$ (A14) and $B=0.60$ (A4). For ${ }_{234}$ these parameters, on assuming that the onset of wave235 growth occurs at $s=0.05$, we find in agreement with observations for the fully developed sea, that from (A5) $u_{10}=3.0 \mathrm{~ms}^{-1}$ and from (A13) $\alpha=0.018$. On using this calibration, we also find that the friction parameter, $R$ (A1) increases from 0.81 at the onset of wave growth $(s=0.05)$ to 1.00 at the maximum in drag coefficient $(s=1)$, and that the wave age, $W$ (A10) increases from 12.5 to 15.4 over the same range.

This set of conditions is a model for the interactions described in Section 2, and the peak wave period at the maximum in drag coefficient $\left(T_{m}\right)$ shows that the full range of sea state conditions which give rise to a maximum in the $10 \mathrm{~m}$ drag coefficient is achievable in the open ocean, and also that at this maximum, the near surface shear is mainly due to the wave motion $(R \approx 1)$. The prediction for the wave generation parameter, $B$, indicates that there is a slip of about 0.6 between the surface wind speed and the peak wave speed which arises from the large scale properties of the storm systems which generate the wave field.

3.2 Sensitivity of the drag law to the peak wave period at the maximum drag coefficient

The relation (A2) shows that $X$ increases as $T_{m}$ increases and $X$ decreases as $T_{m}$ decreases. The $10 \mathrm{~m}$ drag coefficient $K_{10}\left(u_{10}\right)$ derived by interpolation from the inverse drag law (A5) for two widely different maximal peak wave periods, $T_{m}=5 \mathrm{~s}(X=3.89)$ and $T_{m}=50 \mathrm{~s}$ $(X=6.19)$ is shown in Fig. 1 in comparison with that for $T_{m}=17.6 \mathrm{~s}$ discussed in Section 3.1. It is clear that the $10 \mathrm{~m}$ drag coefficient is hardly influenced by these large changes in peak wave conditions over which the Charnock constant, $\alpha$, only varies from 0.016 for $T_{m}=5 \mathrm{~s}$ to 0.019 for $T_{m}=50 \mathrm{~s}$. Hence the theoretical drag law may be said to be almost independent of wave age. From (A4) however, the wave generation parameter has a large variability, ranging from $B=1.41$ at $T_{m}=50 \mathrm{~s}$ to $B=0.22$ at $T_{m}=5 \mathrm{~s}$.

This conclusion indicates that the observed drag law may conceal many possible sea state regimes. A well known regime is the fully developed sea (Toba 1973) in which the wave age,

$W=A$

where $A=1 / 0.029(34.5)$. On the assumption that (10) is applicable at a moderate wind speed, for example $s=0.2\left(u_{\star}=0.36 \mathrm{~ms}^{-1}\right)$, on applying (A10) using (A2), we find that $X=6.1$ and hence $a=0.09 \mathrm{~m}^{-1} \mathrm{~s}$, $B=1.31$ and $R=1.02$. This regime differs from the open ocean regime in so far as the special conditions of a steady constant wind give rise to a regime of reduced spray production (smaller $a$ ) and more efficient wave 


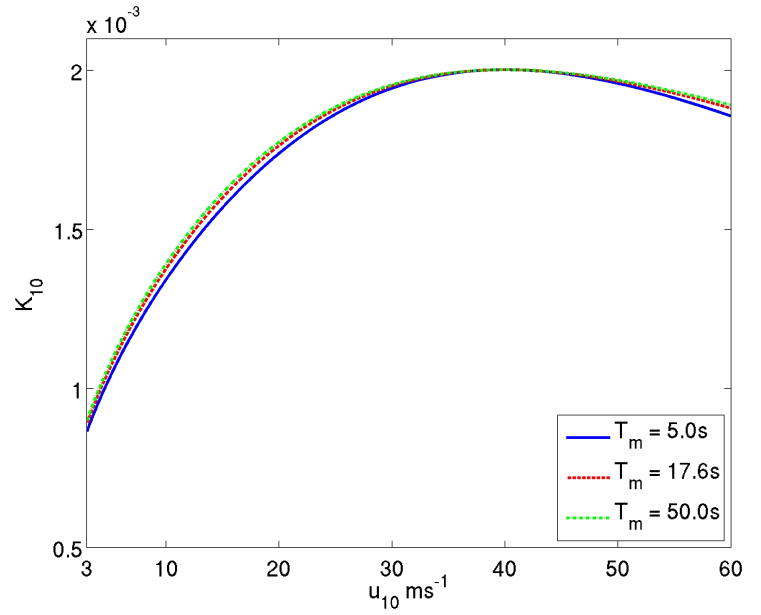

Fig. 1 The theoretical $10 \mathrm{~m}$ drag coefficient, $K_{10}$, as a function of $u_{10}$ derived by interpolation from (A5) for three maximal peak wave periods: (a) $T_{m}=5 \mathrm{~s}$, (b) $T_{m}=17.6 \mathrm{~s}$ and (c) $T_{m}=50 \mathrm{~s}$.

production (larger $B$ ) in which the wave shear dominates the turbulent shear $(R \approx 1)$. This regime however never extends to the maximum in drag coefficient which $_{249}$ from (A10) would occur at a wave period, $T_{m}=46 \mathrm{~s}$, which is not achievable in the ocean. Similar conclusions 250 are found for other moderate values of friction velocity.251 This constant wave age regime was originally discussed $_{252}$ on the assumption that $R=1$ in Bye and Wolff (2004),253 where it was shown that this model was in excellent agreement with observational data (Garratt 1992). $\mathrm{An}^{254}$ important property of this theoretical model for the255 fully developed sea is that from (A9), $\alpha=0.019$ whiche25 is in good agreement with observations (Wu 1980). $\quad{ }_{257}$

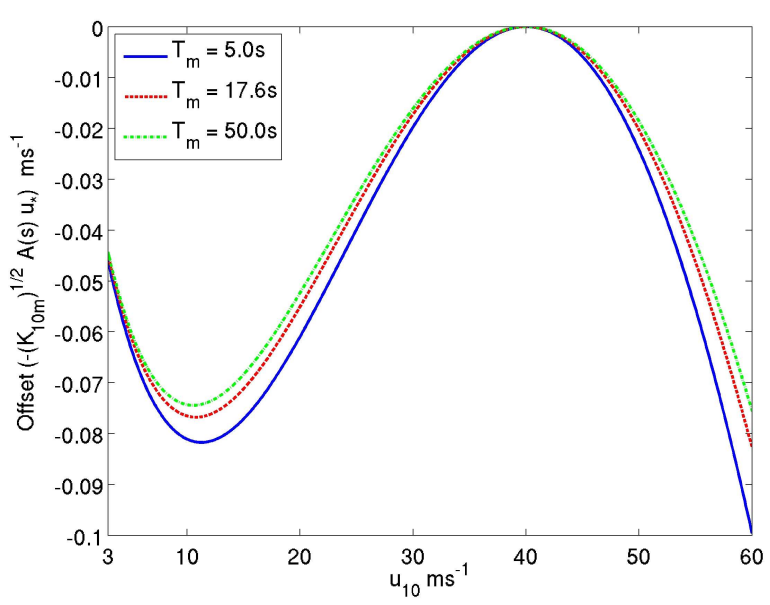

Fig. 2 The offset $\left(-\sqrt{K_{10 m}} A(s) u_{\star}\right)$ of the square root of the ${ }^{274}$ $10 \mathrm{~m}$ drag coefficient (8) as a function of $u_{10}$ for (a) $T_{m}=5^{275}$ $\mathrm{s}$, (b) $T_{m}=17.6 \mathrm{~s}$ and (c) $T_{m}=50 \mathrm{~s}$.

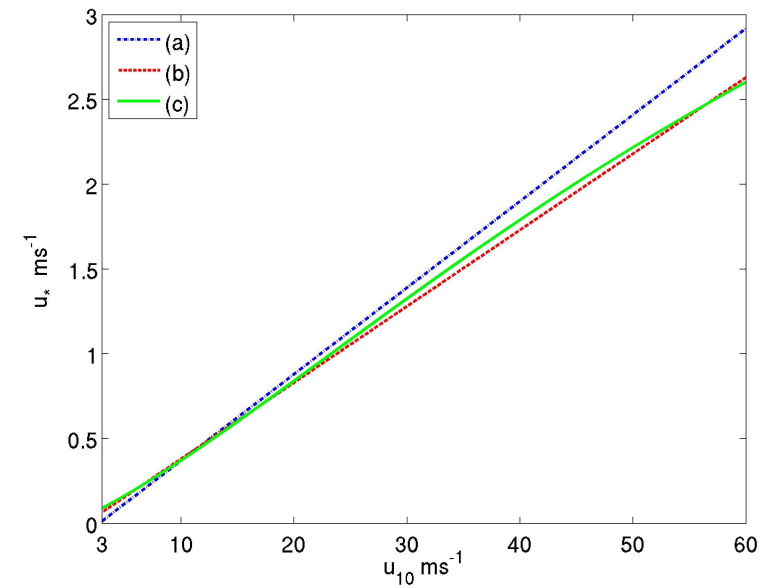

Fig. 3 The friction velocity $\left(u_{\star}\right)$ as a function of the $10 \mathrm{~m}$ wind speed $\left(u_{10}\right)$ : (a) from the linear fit (11) to the observational data, (b) from the linear fit (12) to the theoretical relation (A5), (c) from the theoretical relation (A5) for $T_{m}=17.6$ s.

\section{Comparison with observations}

In a recent analysis of several sets of observations in various locations Foreman and Emeis (2010) proposed that the most appropriate definition of the drag law for the air-sea interface is of the form,

$u_{\star}=C_{m} u_{10}+b$

where $C_{m}$ and $b<0$ are constants. In their analysis of the various data sets the linear relation (11) was obtained by restricting the data to $u_{10} \geq 8 \mathrm{~ms}^{-1}$ which yielded $C_{m}=0.051$ and $b=-0.14 \mathrm{~ms}^{-1}$. On evaluating (11) at $u_{10}=8 \mathrm{~ms}^{-1}, u_{\star}=0.27 \mathrm{~ms}^{-1}$, which the authors claimed to be equal to the onset of aerodynamically rough flow. It is clear from Fig. 2 of Foreman and Emeis (2010) however that the linear relationship extends to a lower friction velocity of about $0.10 \mathrm{~ms}^{-1}$ $\left(u_{10}=5 \mathrm{~ms}^{-1}\right)$ and it also only extends to an upper friction velocity of about $0.85 \mathrm{~ms}^{-1}\left(u_{10}=17 \mathrm{~ms}^{-1}\right)$. At a higher friction velocity $u_{\star}$ tends to become proportional to $u_{10}$ with no offset. In a later more extensive observational study (Andreas et al. 2012) the coefficients of similar linear regressions for $u_{10} \geq 9 \mathrm{~ms}^{-1}$ from aircraft and tower data (778 values) and from a much larger low flying aircraft data set (4878 values) for stable and weakly unstable conditions were respectively $\left(C_{m}=0.0581, b=-0.214 \mathrm{~ms}^{-1}\right)$ and $\left(C_{m}=\right.$ $\left.0.0585, b=-0.243 \mathrm{~ms}^{-1}\right)$.

The evaluation of (8) yields a theoretical value for $C_{m}$ of $0.045\left(K_{10 m}=0.002\right)$, and Fig. 2 shows the offset $\left(-\sqrt{K_{10 m}} A(s) u_{\star}\right)$ as a function of $u_{10}$. Over the range, 
$5 \leq u_{10} \leq 17 \mathrm{~ms}^{-1}$, the offset is almost constant for r $_{30}$ each $T_{m}$ with a mean value of about $-0.07 \mathrm{~ms}^{-1}$, which ${ }_{331}$ is almost independent of $T_{m}$. This range correspond\$332 approximately with that in Foreman and Emeis $(2010)_{333}$ over which (11) is applicable. Hence over this range, the 334 linear relation for the friction velocity derived from (8) is,

$u_{\star}=0.045 \cdot u_{10}-0.07$

which is of the same form as the observed data $(11)_{337}$ although the coefficients in the regression are slightly ${ }_{338}$ different. The theoretical model (Fig. 2) indicates that ${ }_{339}$ the offset decreases at higher $u_{10}<u_{10 m}$ as is indicated ${ }_{340}$ in the observational data, becoming zero at $u_{10 m}$, $\operatorname{and}_{341}$ also decreases at lower $u_{10}$ as is evidenced by (12) which $_{342}$ yields $u_{\star}=0.065 \mathrm{~ms}^{-1}$ at $u_{10}=3.0 \mathrm{~ms}^{-1}$ instead $\mathrm{of}_{343}$ $u_{\star}=0.090 \mathrm{~ms}^{-1}$ from (A5).

Over the mid range of wind speed $\left(5 \leq u_{10} \leq_{345}^{344}\right.$ $17 \mathrm{~ms}^{-1}$ ), Fig. 3 indicates that the linear relation $(12)_{346}$ is very similar to the observational linear relation $(11)_{347}$ of Foreman and Emeis (2010).

Observational estimates of $K_{10 \mathrm{~m}}$ however are incon-349 clusive, see for example Bye et al. (2010), and it is clear ${ }_{350}$ from (8) that a higher value of $K_{10 m}$ would increase ${ }_{351}$ the slope of the theoretical linear regression, e.g. for $_{352}$ $K_{10 m}=0.0025$ the slope would be 0.050 rather than ${ }_{353}$ 0.045 , which is in better agreement with the observa- 354 tions. However, the choice of $K_{10 m}=0.002$ yields $\mathrm{a}_{355}$ Charnock constant at the onset of wave generation, $\alpha={ }_{356}$ 0.018 in good agreement with observations. A higher ${ }_{357}$ value of $K_{10 m}$ would yield a much larger Charnock ${ }_{358}$ constant, and for $u_{10}=40 \mathrm{~ms}^{-1}, T_{m}=17.5 \mathrm{~s}$ and $_{359}$ $K_{10 m}=0.0025, \alpha=0.035$, see (A13). For this reason ${ }_{360}$ we have chosen $K_{10 m}=0.002$ in the evaluation of the ${ }_{361}$ theoretical model, however there is an uncertainty here ${ }_{362}$ that is not yet resolved.

Fig. 3 also shows that the linear relation (12) is in close agreement with the theoretical relation derived ${ }_{364}$ from (A5) for $T_{m}=17.6 \mathrm{~s}$ over the complete range of 365 wind speed from the onset of waves at $3 \mathrm{~ms}^{-1}$ up to $0^{366}$ at least $60 \mathrm{~ms}^{-1}$. There is however an important differ ${ }^{367}$ ence between (12) and (A5). The small scale structure $3_{369}^{368}$ of (A5) shows the occurrence of the maximum in drag ${ }_{370}$ coefficient at $40 \mathrm{~ms}^{-1}$ (Fig. 3), whereas there is no evi-371 dence of this maximum in the fitted linear relation (9).

This is a major problem in the use of linear regressions in defining the drag coefficient at very high wind $_{373}^{372}$ speeds. The high wind speed asymptotic drag coefficient $\left(C_{m}^{2}\right)$ may be much greater than that occurring $9_{374}$ at say $40 \mathrm{~ms}^{-1}$, and the difference depends critically ${ }^{375}$ on the intercept $(b)$ which cannot be determined with $_{377}^{376}$ a high precision due to the extrapolation to a zero $10 \mathrm{~m}^{377}$ wind speed from the wind data set which is truncated ${ }^{378}$ at 8 or $9 \mathrm{~ms}^{-1}$. For the Foreman and Emeis (2010) data set and the two data sets of Andreas et al. (2012), the $40 \mathrm{~m}$ and asymptotic drag coefficients $\left(\times 10^{3}\right)$ are the respective pairs: 2.20 and $2.60,2.78$ and 3.38 , and 2.73 and 3.40 .

\section{Conclusion}

Foreman and Emeis (2010) and Andreas et al. (2012) propose that the linear regression (11) can be applied to the observational data, which is interpreted as a modified drag law in which $b / C_{m}$ is a reference velocity for aerodynamically rough flow governed by a constant drag coefficient $\left(C_{m}^{2}\right)$. The theoretical model shows how the complex interactions which occur in the wave boundary layer give rise to a drag law which can be approximately represented over the range of wind speeds from $3-60 \mathrm{~ms}^{-1}$ by a linear regression of similar form to (11). This result lends strong support to the physical interactions used in the theoretical model as being realistic, however as pointed out in Section 4 the fitting of the linear regression to the observations over a restrictive intermediate range of $u_{10}$ gives rise to uncertainty in the intercept $(b)$ at zero $u_{10}$, and also importantly may miss a maximum in $K_{10}$ at high $u_{10}$, which is important with regard to a main aim of the air-sea boundary layer studies which is to specify the momentum transfer which affects the generation of tropical cyclones and also the mixing below the wave boundary layer which occurs in these extreme events. In summary, the theoretical model, which takes account of the interaction of the three physical processes governing the momentum exchange at the sea surface (Section 2), gives rise to a $10 \mathrm{~m}$ drag law which is almost independent of wave age and is also in agreement with a wide variety of observational data sets.

Acknowledgements JATB gratefully acknowledges the award of a Fellowship at the Hanse-Wissenschaftskolleg, Delmenhorst, Germany in the first half of 2013 during which this study was carried out in the Institute for Chemistry and Biology of the Sea (ICBM), University of Oldenburg. Helpful comments by two referees, especially with regard to the presentation of the theoretical results, are also gratefully acknowledged.

\section{Appendix}

\section{The derivation of the $10 \mathrm{~m}$ drag law}

A convenient analytical procedure is to express the various relations in terms of the non-dimensional parameter $X=$ $1 /\left(a u_{\star m}\right)$. On substituting $X$ in (3) using (6) we obtain the frictional parameter,

$R=\frac{2 \sqrt{K_{I}}}{\kappa} \cdot \frac{X(X-1)}{X-s}$ 
in which $K_{I}$ is the inertial drag coefficient, $\kappa$ is von Kármán's420 constant and $s=u_{\star} / u_{\star m}$, and where from (5) evaluated at ${ }_{421}$ $K_{10 m}$ using (6),

$X=\frac{1}{2}\left[\ln \frac{c_{0 m}^{2}}{2 g z_{10}}+\frac{\kappa}{\sqrt{K_{10 m}}}\right]$

in which $g$ is the acceleration of gravity ilarly, using (2) and (A1), we obtain,

$c_{0}=\frac{(X-1) s}{(X-s)} c_{0 m}$

and the wave generation parameter,

$B=\frac{1}{2} \frac{\kappa c_{0 m}}{X u_{\star m}}$

On substituting for $R$ and $B$ in (5) and using (A2) to $_{432}^{43}$ eliminate $c_{0 m}$, we obtain the following relation for the inverse drag law, $u_{10}\left(u_{\star}\right)$, in terms of $X$,

$u_{10}=\frac{2 u_{\star}}{\kappa}\left\{\frac{X(s-1)}{(X-s)}-\ln \left[\frac{(X-1) s}{(X-s)}\right]\right\}+s u_{10 m}$ (A5)

from which the $10 \mathrm{~m}$ drag law (5) is determined. $X$ can be evaluated from (A2) provided that $K_{10 m}$, and $c_{0 m}$ which depends on sea state, are known. Direct observations of $c_{0 m}$ (or $T_{m}$ ) however are not generally available. It is therefore nec- ${ }^{437}$ essary to use field data at $u_{10}<u_{10 m}$ to infer $X$, as is shown below.

On substituting (A3) in (A2) we obtain,

$X=X^{\prime}+\ln \left[\frac{X-s}{X-1}\right]$ 447

$X=X^{\prime}+\frac{1-s}{X^{\prime}}$

$(\mathrm{A} 7)^{448}$

Hence $X$ may be evaluated in terms of $s, c_{0}$ and $K_{10 m}$ by ${ }^{450}$ substituting $c_{0}$ in $X^{\prime}$.

The Charnock constant can also be evaluated in terms of 452 $X$ and compared with observations. From the defining rela-453 tion for the sea surface roughness $\left(z_{0}\right)$ we have $\ln z_{10} / z_{0}=454$ $\kappa / \sqrt{K_{10}}$ and hence from $(9)$,

$\alpha=\frac{g}{u_{\star}^{2}} z_{10} \exp \left[\frac{-\kappa}{\sqrt{K_{10}}}\right]$

which on eliminating $K_{10}$ using (5) yields,

$\alpha=\frac{1}{2} W^{2} \exp \left[\frac{-R \kappa}{\sqrt{K_{I}}}\right]$

where $W=c_{0} / u_{\star}$ is the wave age (Bye et al. 2010). On substituting for $R$ from (A1), and for $W$ from (A3) which yields,

$W=W_{m} \frac{(X-1)}{(X-s)}$

where $W_{m}=c_{o m} / u_{\star m}$ is the wave age at the drag coefficient maximum, we obtain,

$\alpha=\frac{1}{2} W_{m}^{2}\left[\frac{X-1}{X-s}\right]^{2} \exp \left[-2 X \frac{(X-1)}{(X-s)}\right]$

in which, from (A2),

$W_{m}^{2}=\frac{2 g z_{0}}{u_{\star}^{2}} \exp \left[2 X-\frac{\kappa}{\sqrt{K_{10 m}}}\right]$.
Finally, on substituting (A12) in (A11) we have the expression for the Charnock constant,

$\alpha=\left[\frac{X-1}{X-s}\right]^{2} \frac{g z_{10}}{u_{\star m}^{2}} \exp \left[2 \frac{X(1-s)}{(X-s)}\right] \exp \left[\frac{-\kappa}{\sqrt{K_{10 m}}}\right]$

We note that (A13), which is independent of $c_{0 m}$, indicates that the choice of $K_{10 m}$, rather than $u_{\star m}$ or $c_{0 m}$, is highly significant in the evaluation of $\alpha$. In terms of $X$, the spray parameter,

$a=\frac{1}{X u_{\star}}$

These expressions will be evaluated using the observed estimates at the maximum drag coefficient of $K_{10 m}=0.002$ and $u_{10 m}=40 \mathrm{~ms}^{-1}\left(u_{\star m}=1.79 \mathrm{~ms}^{-1}\right)$, and also $\kappa=0.4$, $g=9.8 \mathrm{~ms}^{-2}$ and $K_{I}=0.0015$, and at the onset of wave growth $(s=0.05)$ yield from (A5) $u_{10}=3.0 \mathrm{~ms}^{-1}$, and, $\alpha=0.018$ in agreement with observations for the fully developed sea (Section 3.2). Note that all these expressions, except for (A1) and (A9) which involve $R$, are independent of $K_{I}$.

\section{References}

Andreas EL, Mahrt L, Vickers D (2012) A new drag relation for aerodynamically rough flow over the ocean. J Atmos Sci 69 2520-2537

Bye JAT, Babanin AV (2009) Wave generation by wind. Encyclopedia of Ocean Sciences (Second Edition). Steele JH, Turekian KK, Thorpe SA (eds) 304-309

Bye JAT, Ghantous MP, Wolff J-O (2010) On the variability of the Charnock constant and the functional dependence of the drag coefficient on wind speed. Ocean Dyn 60 851-860

Bye JAT, Wolff J-O (2004) Prediction of the drag law for air-sea momentum exchange. Ocean Dyn 54 577-580

Bye JAT, Wolff J-O (2008) Charnock dynamics: a model for the velocity structure in the wave boundary layer of the air-sea interface. Ocean Dyn 58 31-42

Foreman RJ, Emeis S (2010) Revisiting the definition of the drag coefficient in the marine atmospheric boundary layer. J Phys Oceanogr 402325 - 2332

Garratt JR (1992) The atmospheric boundary layer. Cambridge University Press, Cambridge 307 pp

Toba Y (1973) Local balance in the air-sea boundary process III. On the spectrum of wind waves. J Oceanogr Soc Japan 29 209-220

Wu J (1980) Wind-stress coefficients over sea surface nearneutral conditions - a revisit. J Phys Oceanogr 10 727-740 


\section{University Library}

\section{- M M N E R VA A gateway to Melbourne's research publications}

Minerva Access is the Institutional Repository of The University of Melbourne

Author/s:

Bye, JAT;Wolff, J-O;Lettmann, KA

Title:

On the variability of the Charnock constant and the functional dependence of the drag coefficient on wind speed: Part II-Observations

Date:

2014-07-01

Citation:

Bye, J. A. T., Wolff, J. -O. \& Lettmann, K. A. (2014). On the variability of the Charnock constant and the functional dependence of the drag coefficient on wind speed: Part II-Observations. OCEAN DYNAMICS, 64 (7), pp.969-974. https://doi.org/10.1007/ s10236-014-0735-4.

Persistent Link:

http://hdl.handle.net/11343/282842 